\title{
@s \\ Origin of incommensurate modulations in the high-pressure phosphorus IV phase
}

\author{
M. Marqués, G. J. Ackland, L. F. Lundegaard, S. Falconi, C. Hejny, and M. I. McMahon* \\ SUPA, School of Physics and Centre for Science at Extreme Conditions, The University of Edinburgh, \\ Mayfield Road, Edinburgh EH9 3JZ, United Kingdom \\ J. Contreras-García \\ Departamento de Química Física y Analítica and MALTA-Consolider, Universidad de Oviedo, E-33006 Oviedo, Spain \\ M. Hanfland \\ European Synchrotron Radiation Facility, BP 220, 38043 Grenoble, Cedex, France
}

(Received 13 February 2008; revised manuscript received 16 June 2008; published 22 August 2008)

\begin{abstract}
We present results from $\mathrm{x}$-ray diffraction experiments and density functional theory calculations which provide a fully consistent picture of the high-pressure phosphorus-IV phase (P-IV). P-IV has an incommensurately-modulated crystal structure described by the four-dimensional superspace group $\mathrm{Cmmm}(00 \gamma) s 00$. Electronic structure calculations using a three-dimensional commensurate approximant to this structure give excellent agreement with experiment for the structural parameters and their variation with pressure. Density functional perturbation theory shows a phonon instability at the incommensurate wave vector, related to the opening of a pseudogap at the Fermi surface, showing that the atomic motions comprising the incommensuration occur to eliminate Fermi-surface nesting. Unusually, the pseudogap opens not at the incommensurate wave vector $(00 \gamma)$ itself, but at two larger wave vectors corresponding to strong reflections in the diffraction pattern.
\end{abstract}

DOI: 10.1103/PhysRevB.78.054120

PACS number(s): 61.50.Ks, 71.20.-b, 62.50.-p

\section{INTRODUCTION}

Since their discovery in the group-I and -II metals, complex and incommensurately-modulated phases have been observed in a wide variety of elements at high pressure. ${ }^{1,2}$ The early theoretical picture of the appearance of complex phases at high pressure was that they arose due to the complicated structure of $d$-orbitals occupied under pressure by $s-d$ electron transfer. This explanation, however, is incompatible with the recent discovery of complex phases at intermediate pressures in lithium, sulfur, selenium, and tellurium. ${ }^{1,2}$ In most cases, the picture of structural complexity arising from the opening of a pseudogap at the Fermi level due to Fermi surface-Brillouin zone (FSBZ) interactions appears to be more relevant, ${ }^{3}$ and unifies the picture with that found in tetravalent materials, ${ }^{4}$ and even high-pressure liquids. 5,6

A recent addition to the list of complex high-pressure phases, and, in particular, to those such phases which are stable above $100 \mathrm{GPa}$, is that of the "intermediate" phase of phosphorus, P-IV, which is stable between $107 \mathrm{GPa}$ and 137 $\mathrm{GPa}^{7}$ Long thought to have a complex structure, ${ }^{7}$ it has recently attracted a number of computational studies ${ }^{8-11}$ which, using full potential linear muffin-tin orbital (FPLMTO) and the frozen core all-electron projector augmented wave methods, have proposed a number of different structures: an orthorhombic Imma structure with atoms on $4 e$ sites, ${ }^{8}$ a hostguest Ba-IV type structure, ${ }^{9}$ and, most recently, an orthorhombic $C 2 \mathrm{~mm}$ structure with atoms on $2 a$ and $2 b$ sites. $^{10}$ Metadynamics simulations ${ }^{11}$ have also concluded that the structure is monoclinic, but with the atoms possessing a modulated pattern.

Very recently, experimental studies of P-IV have reported it as having a previously unobserved incommensurately- modulated structure, ${ }^{12}$ with an atomic arrangement very similar to that proposed in the metadynamics simulation. ${ }^{11}$ Perhaps the most striking thing about the incommensurate structure of P-IV is the very large amplitude of the modulation, which results in atomic displacements some $15 \%$ of the unit-cell length, approximately $0.4 \AA$, much larger even than that observed previously in Te-III at the much lower pressure of $5 \mathrm{GPa} .{ }^{13} \mathrm{P}-\mathrm{IV}$ is also unique among the incommensurate elements in that the strongly modulated phase lies between two phases with extremely simple crystal structures-simple cubic up to $107 \mathrm{GPa}$, and simple hexagonal above $137 \mathrm{GPa}^{7}$ While the average structure of the incommensurate phase can be seen to be intermediate between those of the two much simpler structures, ${ }^{12}$ previous studies cast no insight into why the structure should be modulated. In this paper we present a consistent picture of P-IV, based on detailed $\mathrm{X}$-ray powderdiffraction studies of the pressure dependence of its structure and transitions, density functional theory (DFT) calculations of its structural stability, and a theoretical examination of the incommensuration as a phonon softening of a putative undistorted phase.

\section{X-RAY DIFFRACTION STUDY OF P-IV}

Powder-diffraction data were collected on station ID09a at the European Synchrotron Radiation Facility (ESRF), Grenoble, using an incident $x$-ray wavelength of $\sim 0.41 \AA$ and a beam size of $10 \mu \mathrm{m} \times 10 \mu \mathrm{m}$. The powdered sample of orthorhombic black-P was provided by U. Schwarz (Dresden) and was loaded into two diamond-anvil cells equipped with bevelled diamonds and with either $\mathrm{He}$ or $\mathrm{H}_{2}$ as a pressure transmitting medium. A third pressure cell, also 


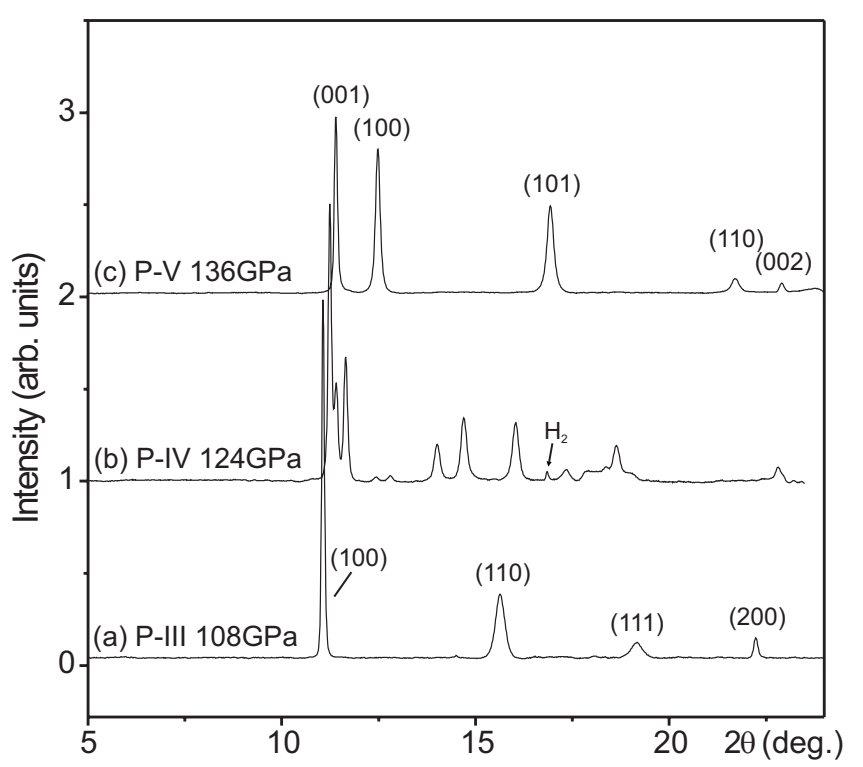

FIG. 1. Diffraction profiles from (a) simple cubic P-III at 108 $\mathrm{GPa}$, (b) intermediate phase P-IV at $124 \mathrm{GPa}$ and (c) simple hexagonal $\mathrm{P}-\mathrm{V}$ at $136 \mathrm{GPa}$. The background arising from Compton scattering in the diamond anvils has been subtracted from all three profiles. The additional sharp peak in profile (b) is from the $\mathrm{H}_{2}$ pressure transmitting medium.

equipped with bevelled anvils, was loaded with no pressure medium. A few grains of polycrystalline tantalum (grain size 2-3 $\mu \mathrm{m}$ ) were enclosed with the samples for pressure measurement via the Ta equation of state. ${ }^{14}$ The sharpest diffraction peaks were obtained from the sample enclosed in the $\mathrm{H}_{2}$ pressure medium, and it was therefore with this sample that the majority of the diffraction data were obtained. The diffraction data were collected on an image-plate detector placed approximately $367 \mathrm{~mm}$ from the sample. To improve powder averaging, the sample was oscillated by $\pm 3^{\circ}$ during the $60 \mathrm{~s}$ exposures. The resulting two-dimensional diffraction images were integrated using FIT2D (Ref. 15) to give standard diffraction profiles. Rietveld refinement of these profiles was performed using the JANA2000 software package. ${ }^{16}$

Upon pressure increase, diffraction patterns from the simple cubic (SC) phase were observed up to 110(2) GPa, at which point additional peaks from the intermediate phase were observed. Further pressure increase resulted in the disappearance of all the SC peaks, and single-phase patterns of the intermediate phase were observed up to 131(1) GPa, at which pressure diffraction peaks from the simple hexagonal (SH) phase appeared. These remained up to $139 \mathrm{GPa}$, the highest pressure reached in this study. Diffraction profiles from single-phase samples of the three different phases are shown in Fig. 1.

The diffraction pattern from the intermediate phase is the same as that reported by Fujihisa et al., ${ }^{12}$ and all the diffraction peaks can be accounted for by the modulated structure they propose, that is, superspace group $\mathrm{Cmmm}(00 \gamma) s 00$ with a $\mathrm{P}$ atom located on the $2 a$ site at $(0,0,0)$. At $124 \mathrm{GPa}$, the orthorhombic lattice parameters are $a_{o}=2.766(1) \AA, \quad b_{o}=3.204(1) \AA$ and $c_{o}=2.064(1) \AA$, atomic volume 9.146(4) $\AA^{3}$, with $\gamma=0.267(1)$ and a modu-

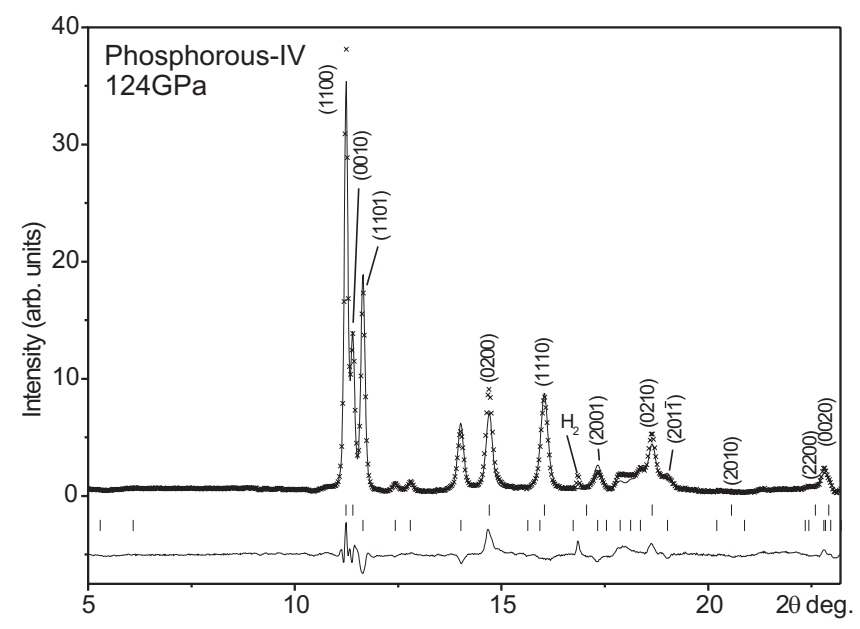

FIG. 2. Rietveld refinement of P-IV at $124 \mathrm{GPa}$. The upper and lower tick marks below the profile show the peak positions of the main and satellite reflections, respectively. The indices of some of the Bragg peaks are given. The Bragg peak from the $\mathrm{H}_{2}$ pressure medium is also indicated.

lation amplitude $B_{1 x}=0.159(1)$. All parameters are in excellent agreement with those reported by Fujihisa et al. at the very similar pressure of $125 \mathrm{GPa}^{12} \mathrm{~A}$ Rietveld refinement of incommensurate P-IV at $124 \mathrm{GPa}$ is shown in Fig. 2, and the pressure dependence of the atomic volume is shown in Fig. 3 . The relative volume changes $\left(\Delta V / \mathrm{V}_{\text {trans }}\right)$ at the $\mathrm{III} \rightarrow \mathrm{IV}$ and $\mathrm{IV} \rightarrow \mathrm{V}$ at $110(2)$ and $131(1) \mathrm{GPa}$ are $1.4(1) \%$ and $4.5(1) \%$, respectively, both some $\sim 1 \%$ smaller than the values reported by Fujihisa et al. ${ }^{12}$ The inset to Fig. 3 shows the pressure dependence of the incommensurate wave vector $(00 \gamma)$, which is independent of pressure across the stability range of P-IV. Refinements of the modulation amplitude showed that it increases with pressure, in agreement with the behavior reported by Fujihisa et al., from $0.132(1)$ to

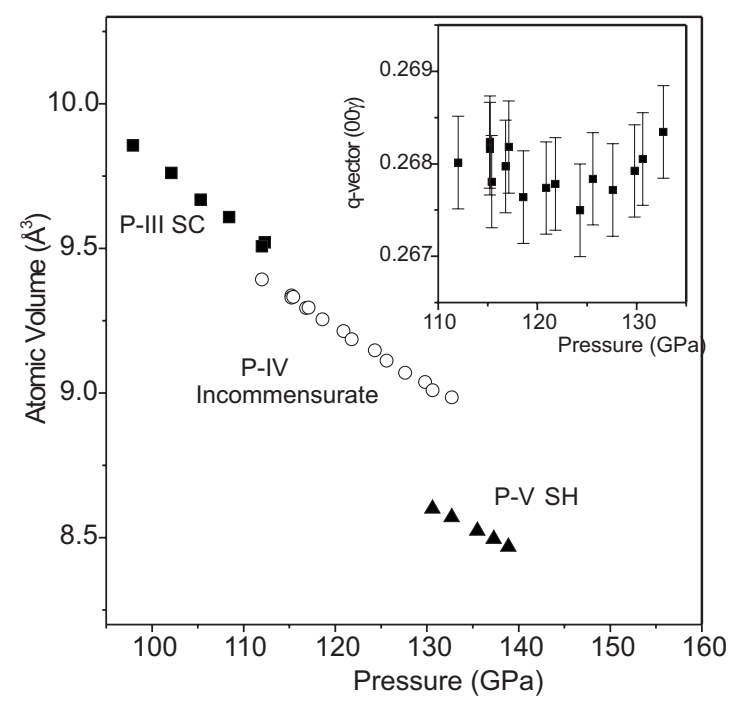

FIG. 3. Pressure dependence of the atomic volume of SC P-III, incommensurate P-IV and SH P-V, showing the first-order nature of both the III-IV and IV-V transitions. The inset shows the pressure dependence of the incommensurate wave vector $(00 \gamma)$. 
(a)

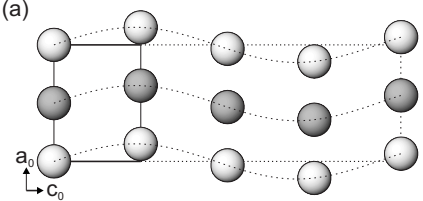

(b)

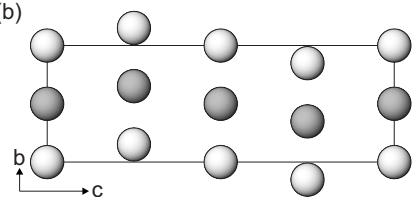

(c)

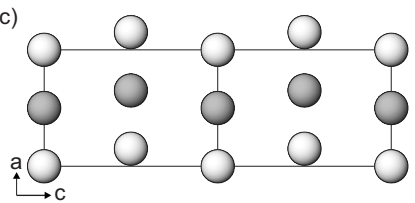

FIG. 4. (a) Four unit cells of the incommensurately-modulated structure of P-IV as seen looking down the $b$ axis. The dotted line shows the modulation wave. (b) Structure of the $\mathrm{Cmcm}$ and (c) $C 2 \mathrm{~mm}$ approximants shown in $a$-axis and $b$-axis projections, respectively. Two unit cells of the $C 2 \mathrm{~mm}$ approximant are shown for ease of comparison with the $\mathrm{Cmcm}$ approximant. In all three structures, the dark gray atoms are located at a height of (a and c) $y$ $=\frac{1}{2}$ or $(\mathrm{b}) x=\frac{1}{2}$.

$0.159(1)$ over the pressure range $115 \mathrm{GPa}$ to $130 \mathrm{GPa}$. This behavior is in marked contrast to the incommensurate modulations in $\mathrm{Se}$ and $\mathrm{Te}$ which decrease rapidly with pressure. $^{13,17}$

Our experimental data thus fully corroborate both the recent report of Fujihisa et al. $^{12}$ and the metadynamics simulations of Ishikawa et al.: ${ }^{11} \mathrm{P}-\mathrm{IV}$ is incommensurate with a large, almost pressure independent, modulation.

\section{COMMENSURATE APPROXIMATIONS OF P-IV}

The description of incommensurate structures is a major problem for first-principles electronic structure calculations, which are limited to periodic structures as a consequence of employing periodic boundary conditions. Calculations usually involve approximant cells trying to describe the real physics of the incommensurate cell. In phosphorus, since $\frac{1}{\gamma}$ $=3.74$, phase IV is best approximated by a rational approxi- mant corresponding to $\gamma=\frac{1}{4}$, and a quadrupling of the unit cell along the $c$ axis. The resulting commensurate structure has space group $C m c m$ with $a_{C m c m}=b_{o}, b_{C m c m}=a_{o}, c_{C m c m}$ $=4 c_{o}$ and with two $\mathrm{P}$ atoms on the $4 a(0,0,0)$ and $4 c\left(0, y, \frac{1}{4}\right)$ sites [see Fig. 4(b)]. The $y$ coordinate of the atom on the $4 c$ position is 0.159 , the same as the $B_{1 x}$ modulation amplitude in the incommensurate description of the structure. It should be noted that the eight-atom-cell $\mathrm{Cmcm}$ approximant corresponds to a particular choice (locked in) of phase in the modulation wave that does not affect the definition of the incommensuration.

While this eight-atom $\mathrm{Cmcm}$ structure is the best commensurate approximant to the true incommensurate structure, previously proposed structures for P-IV can also be observed to be (poorer) approximants. The orthorhombic Imma structure of Ahuja ${ }^{8}$ corresponds to a distortion of the unmodulated $\mathrm{Cmmm}$ structure according to the irreducible representation T3- (associated with a doubling of the $c$ axis). The unit cell of the Imma structure is then $2 c_{o} \times b_{o} \times a_{o}$, and hence involves a doubling (rather than a quadrupling) of the incommensurate unit cell. However, the Imma structure does not include the atomic displacements required to model the modulation.

A better approximant is given by the orthorhombic $C 2 \mathrm{~mm}$ structure of Mikhaylushkin et al., ${ }^{10}$ where, using their computed axial ratios and our experimental atomic volume of $9.15 \AA^{3}$ at $124 \mathrm{GPa}$, the lattice parameters would be $a_{C 2 \mathrm{~mm}}$ $=2.753 \AA, b_{C 2 m m}=3.220 \AA$, and $c_{C 2 m m}=4.129 \AA$. These are very close to experimental values at the same pressure (see Sec. II above), with a doubling of the $c_{o}$ axis. The $C 2 \mathrm{~mm}$ approximant [see Fig. 4(c)] also incorporates the displacement of successive atomic planes along the incommensurate direction, but, because of the doubling (rather than quadrupling) of the $c_{o}$ axis, the modulation is zig-zag-zig-zag, rather than the correct zig-zag-zag-zig displacements of the $\mathrm{Cmcm}$ approximant. However, the amplitude of the displacement of successive layers of 0.15 is close to the experimental value.

As space group $C 2 \mathrm{~mm}$ is a subgroup of $\mathrm{Cmcm}$, both structures can be described using the $C 2 \mathrm{~mm}$ subgroup- or Amm 2 in its standard setting. The relationship between the two descriptions is given in Table I.

\section{METHOD}

First-principles total-energy calculations were carried out within the DFT formalism with a plane-wave pseudopoten-

TABLE I. The SC, SH, average $\mathrm{Cmmm}, \mathrm{Cmcm}$ approximant and $\mathrm{C} 2 \mathrm{~mm}$ approximant structures of phosphorus, as described in a common orthorhomic structure with space group Amm2.

\begin{tabular}{lccccc}
\hline \hline & SC $(P m \overline{3} m)$ & SH $(P 6 / m m m)$ & orth1 $(\mathrm{Cmmm})$ & orth2 (Cmcm) & orth3 $(\mathrm{C} 2 \mathrm{~mm})$ \\
& $a_{c}$ & $a_{h}, c_{h}$ & $a_{o}, b_{o}, c_{o}$ & $a, b, c, y$ & $a_{a}, b_{a}, c_{a}, x_{1}, x_{2}$ \\
\hline$a_{S}$ & $4 a_{c}$ & $4 c_{h}$ & $4 c_{o}$ & $c$ & $2 c_{a}$ \\
$b_{S}$ & $\sqrt{2} a_{c}$ & $\sqrt{3} a_{h}$ & $b_{o}$ & $a$ & $b_{a}$ \\
$c_{S}$ & $\sqrt{2} a_{c}$ & $a_{h}$ & $a_{o}$ & $b$ & $a_{a}$ \\
$P(4 c)$ & $\left(\frac{1}{4}, 0,0\right)$ & $\left(\frac{1}{4}, 0,0\right)$ & $\left(\frac{1}{4}, 0,0\right)$ & $\left(\frac{1}{4}, 0,0\right)$ & $\left(\frac{1}{4}, 0, x_{1}\right)$ \\
$P(2 a)$ & $(0,0,0)$ & $(0,0,0)$ & $(0,0,0)$ & $(0,0, y)$ & $\left(0,0, x_{2}\right)$ \\
$P(2 b)$ & $\left(\frac{1}{2}, 0,0\right)$ & $\left(\frac{1}{2}, 0,0\right)$ & $\left(\frac{1}{2}, 0,0\right)$ & $\left(\frac{1}{2}, 0,-y\right)$ & $\left(\frac{1}{2}, 0, x_{2}\right)$ \\
\hline \hline
\end{tabular}


tial approach, as implemented in the Vienna ab initio simulation package (VASP). ${ }^{18}$ We used the Perdew-Wang generalized gradient approximation (GGA) exchange-correlation functional $^{19}$ and the projector augmented wave (PAW) allelectron description of the electron-ion-core interaction. ${ }^{20}$ Brillouin-zone integrals were approximated using the method of Monkhorst and Pack, ${ }^{21}$ and the energies converged to $1 \mathrm{meV}$ with respect to $k$-point density and $0.2 \mathrm{meV}$ with respect to plane-wave cutoff $(319 \mathrm{eV})$. For each lattice we calculated the total energy per atom $(E)$ at a number of selected values of the volume per atom $(V)$, relaxing the atomic coordinates and lattice parameters subject to the constraints of symmetry and volume conservation. All structural relaxations were performed via a conjugate-gradient minimization of the total energy using the Methfessel-Paxton method. For the final calculation of the optimized crystal structures the tetrahedron method with Blöchl correction was used. The variation with hydrostatic pressure of the lattice parameters and atomic coordinates has been obtained by means of numerical and standard equations of state fittings to the sets of computed $(E, V)$ points. ${ }^{22}$ This procedure also provides $G(p)$ curves in the static approximation (zero temperature and neglecting zero-point vibrational contributions).

Phonon frequencies were calculated within densityfunctional perturbation theory ${ }^{23}$ as implemented in the QUANTUM-ESPRESSO code. ${ }^{24}$ The total energies required in the calculation of the phonons were obtained using the same Perdew-Wang parametrization of the exchange-correlation potential. The ion-electron-interaction was described by an ultrasoft pseudopotential with nonlinear core corrections generated according to the Vanderbilt scheme. ${ }^{25}$ The electronic wave function and the charge density were expanded with a kinetic energy cutoff of 408.17 and $4081.71 \mathrm{eV}$, respectively. Brillouin-zone integrations were performed using the same grids as in the VASP calculations.

To compute the full phonon dispersion curves, we computed the interatomic force constants by Fourier transformation of the dynamical matrices computed on $(8,8,8)$ and $(6,6,6)$ regular $q$-point grids. It is important to note that the description of the incommensuration requires a much finer mesh along the direction where the incommensuration appears. Due to the fact that in metallic systems the direct computation of the dynamical matrix at a specific $q$ point requires its inclusion in the $k$-point grid, i.e., $k=q+k^{\prime}$, it demands finer $k$-point sampling of the Brillouin zone.

Finally, the topological analysis of the electron localization function (ELF) ${ }^{26,27}$ was performed using the CRITIC program with the aim of characterizing the chemical bonding. ${ }^{28}$ To this end, the VASP optimized structures were recalculated with the CRYSTAL9 8 code $^{29}$ using the same exchange and correlation functionals as in the pseudopotential calculations in order to obtain all electron wave functions, required for a reliable and quantitative analysis of the ELF topology. It is interesting to remark that the topology induced by the ELF field allows a partition of the three-dimensional space into nonoverlapping basins associated with the maxima (or attractors) of the ELF and surrounded by ELF zero flux surfaces. These basins have a full chemical meaning within the valence shell electron pair repulsion (VSEPR): atomic shells, bonds and lone pairs. This ability makes the ELF an appro-

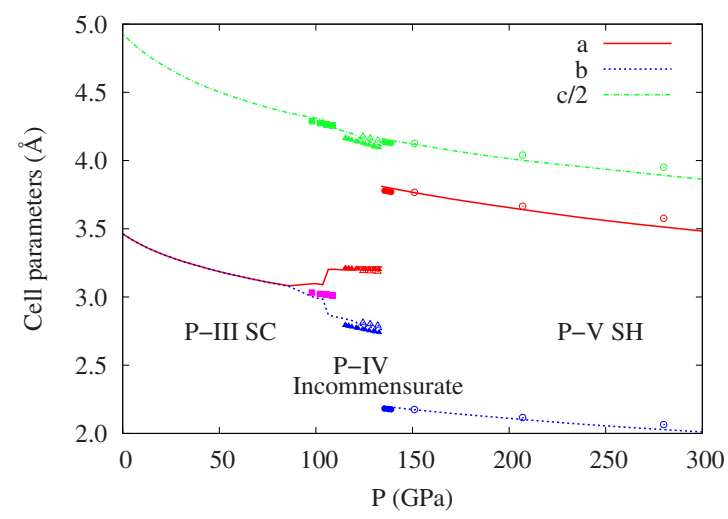

FIG. 5. (Color online). Calculated cell parameters of the SC, $\mathrm{SH}$, and $\mathrm{Cmcm}$ approximant phases, all calculated using the $\mathrm{Cmcm}$ structure (lines). The solid and open symbols show our, and previous (Ref. 30), experimental data, respectively. Also shown are the calculated cell parameters at selected pressures (open triangles) obtained using the $C 2 \mathrm{~mm}$ approximant of Mikhaylushkin et al. (Ref. $10)$.

priate formalism to reconcile the traditional chemical language with the outcome of quantum-mechanical calculations. The CRITIC code is able to completely characterize the ELF topology through the localization of all the critical points and the determination of the limiting surfaces which define the basins. Hence, the integrations within these basins provide charges and volumes of atomic shells, bonds and lone pairs.

\section{RESULTS}

\section{A. Total-energy calculations}

The evolution of the calculated cell parameters with pressure for the SC P-III, incommensurate P-IV (using the $\mathrm{Cmcm}$ approximant) and the SH P-V phases, along with the experimental data, is shown in Fig. 5. To minimize sampling errors all three structures were described using the eight-atom Cmcm structure, which, in the case of SC and $\mathrm{SH}$, has $y_{P(4 c)}=0$ and is therefore unmodulated. The transformations of the cell parameters and atomic coordinates from the conventional SC and SH cells, the unmodulated Cmmm cell and the eight-atom $\mathrm{Cmcm}$ and four-atom $\mathrm{C} 2 \mathrm{~mm}$ approximants to a common orthorhombic cell that contains eight atoms and belongs to the Amm2 space group (the conventional setting of the $C 2 \mathrm{~mm}$ space group) are given in Table I.

As can be seen in Fig. 5, good agreement is found between theory and experiment, especially for the SC and SH phases. It should also be noted that optimization of the unmodulated Cmmm structure resulted in it always reverting back to the $\mathrm{SC}$ or $\mathrm{SH}$ structures, depending on the volume considered. For the incommensurate phase, the $\mathrm{Cmcm}$ approximant calculation leads to an overestimation of the volume by about $2 \%$ with respect to the experimental value as a consequence of $\sim 0.8 \%$ and $\sim 0.6 \%$ bigger $b$ and $c$ cell parameters. The reason for this comes from the nature of the incommensuration, in which planes of atoms perpendicular to the incommensurate $c$ axis are displaced along the $\pm b$ axis. Interestingly, and as a consequence of the similar local 


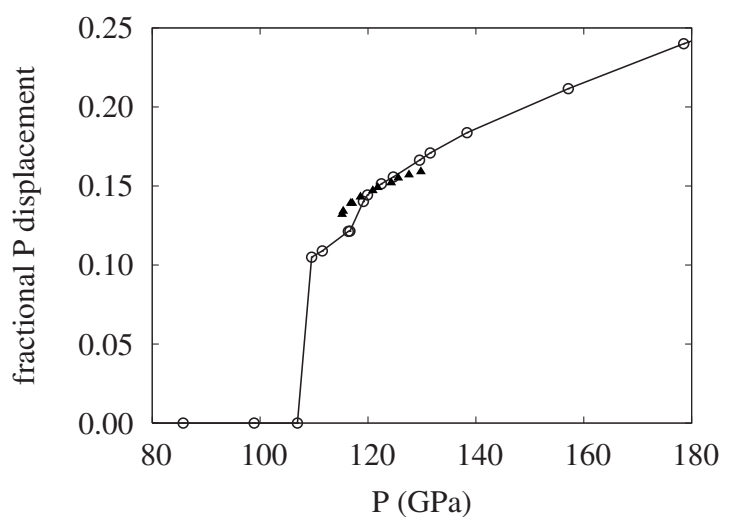

FIG. 6. Calculated pressure dependence of the modulation amplitude in P-IV. The open and closed symbols show our calculated and experimental data, respectively.

environments, structural optimization of the $C 2 \mathrm{~mm}$ structure proposed by Ref. 10 at selected pressures also showed good agreement with the experimental values, having, however, slightly bigger discrepancies for the $b$ and $c$ axes that led to a poorer description of the incommensuration. As a consequence, the $\mathrm{Cmcm}$ approximant has lower enthalpy and is the most favored structure among those investigated.

Calculated values of the modulation amplitude in P-IV, as obtained from the fractional displacement of the $\mathrm{P}(4 c)$ atom along the $b$ axis, are shown in Fig. 6. It can be seen that the modulation amplitude increases with pressure, in agreement with the experimental data. The calculated enthalpies of the $\mathrm{SC}, \mathrm{SH}$, and $\mathrm{Cmcm}$ approximant phases as a function of pressure are shown in Fig. 7. The stability range of the $\mathrm{Cmcm}$ approximant structure is $108 \mathrm{GPa}-136 \mathrm{GPa}$, in excellent agreement with the experimental values of 110(2) GPa to 131(1) GPa for the incommensurate phase.

\section{B. Dynamical stability}

Since the incommensurate phase can be formed by symmetry breaking, it is interesting to analyze the phonon dispersion curves of both the SC and the SH structures with increasing and decreasing pressure, respectively, in order to

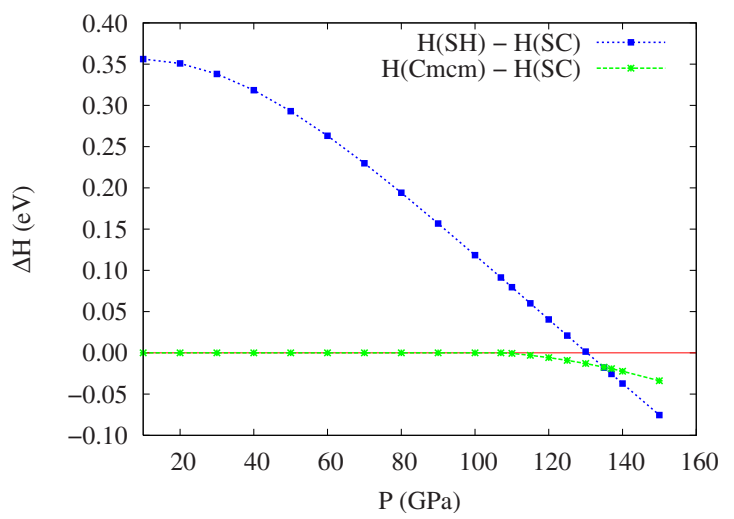

FIG. 7. (Color online) Calculated pressure dependence of the enthalpies of the $\mathrm{Cmcm}$ and $\mathrm{SH}$ phases relative to that of the SC phase.
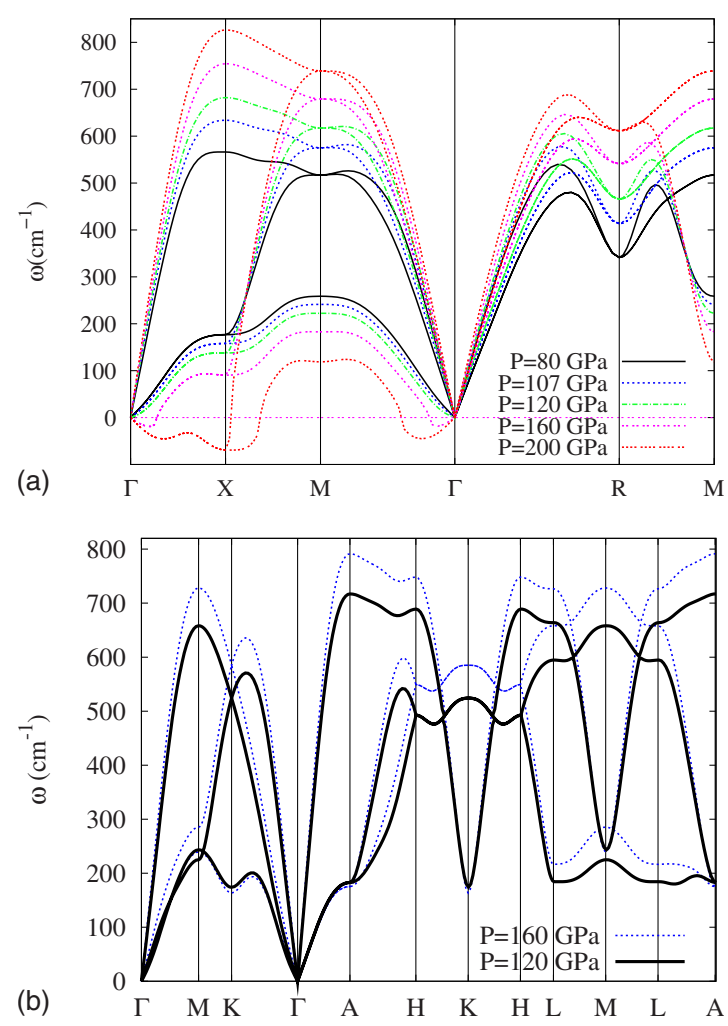

FIG. 8. (Color online) Calculated phonon dispersion curves for (a) the $\mathrm{SC}$ and (b) the $\mathrm{SH}$ structures versus pressure.

detect any instability associated with the transition to the incommensurate phase. From group-theoretical analysis, ${ }^{31}$ $\mathrm{Cmmm}(00 \gamma) s 00$ arises from the irreducible representation (IR) $\Delta_{5}$ associated with the $\mathbf{k}$ vector $\Delta=(0, \beta, 0)$ (in the $\Gamma-X$ direction) and from the IR $\Delta_{6}$ associated with the $\mathbf{k}$ vector $\Delta=(0,0, \mathrm{~g})$ (in the $\Gamma-A$ direction) for the $\mathrm{SC}$ and $\mathrm{SH}$ phases, respectively.

For the SC structure, we observe (Fig. 8) a softening of the lowest-frequency transverse mode along the directions $\Gamma-X-M-\Gamma$ (in the $\Gamma-X$ direction this mode is twofolddegenerate corresponding then to both transverse branches) when going from 80 to $120 \mathrm{GPa}$. At $140 \mathrm{GPa}$, the transverse acoustic (TA) frequencies become imaginary near the zone center. An independent calculation of the elastic constants confirms that this is related to the elastic constant $C_{44}$ becoming negative at $140 \mathrm{GPa}$. At even higher pressure (200 GPa) we observe that the whole acoustic branch becomes imaginary along the $\Gamma-X$ direction, with two minima (one close to the incommensurate wave vector, the other to the $X$ point). Similarly, there is an instability in the $\Gamma-M$ direction, which moves toward $M$ with increasing pressure. A finer $q$-point $(2 \times 2 \times 48)$ sampling along the $\Gamma-X$ direction confirms the presence of two minima at $\sim 0.3$ of the $\Gamma X$ distance and at the $X$ point. According to the group-theoretical analysis these could be related to the observed incommensuration of the P-IV phase.

In the SH phase, on the other hand, upon pressure decrease from $160 \mathrm{GPa}$ to $120 \mathrm{GPa}$, we do not observe any phonon anomaly. At lower pressures ( $\sim 80 \mathrm{GPa})$ imaginary frequencies appear at the $M$ point and along the $\Gamma-K$ line, but, according to the group-theoretical analysis, these cannot 

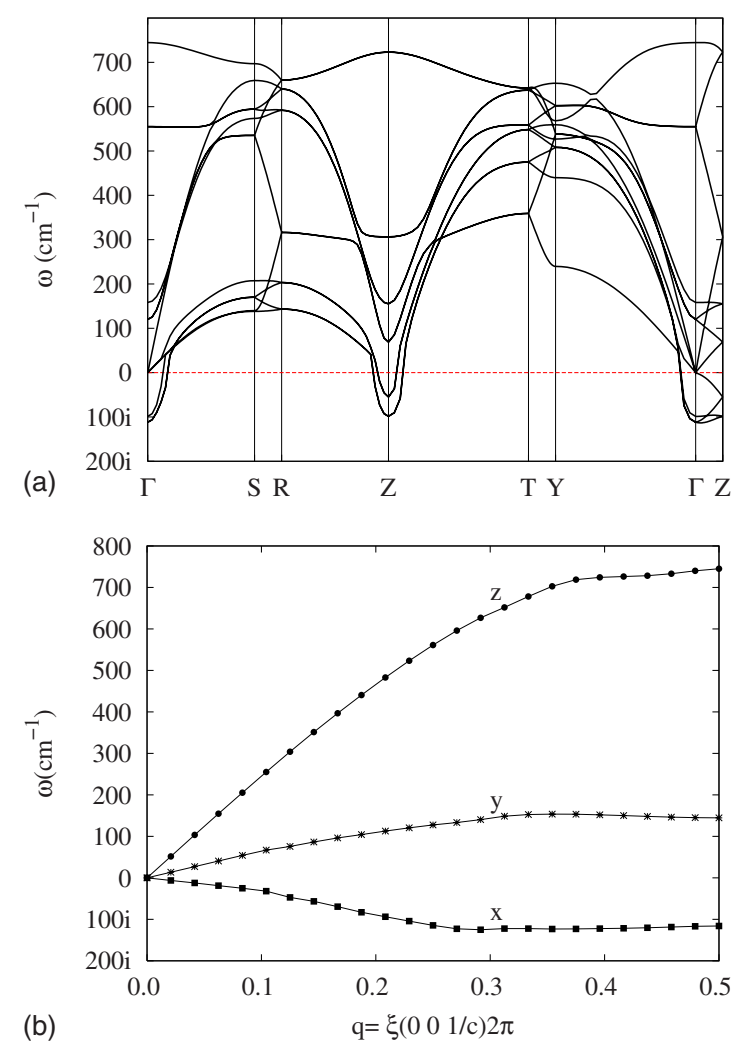

FIG. 9. (Color online) (a) Calculated folded phonon dispersion of unmodulated $\mathrm{Cmcm}$ and (b) phonon dispersion of the $\mathrm{Cmmm}$ structure along the $\Gamma-Z$ direction.

be related to the observed incommensuration.

It should be noted that the geometry of the SCincommensurate transformation is not simply a pure phonon (defined as atomic displacements in an unchanged unit cell). Rather, the transition involves a distortive coupling of the phonon to a changed lattice parameter. In order to decouple these two effects, and thereby investigate the effects of phonons alone, we have also investigated the dynamics of the unmodulated $\mathrm{Cmmm}$ structure.

Under geometry optimization, the average $\mathrm{Cmmm}$ structure reverts back to the SC structure at pressures below 108 $\mathrm{GPa}$. In order to investigate the dynamics of the incommensurate phase, we examined the commensurate $\mathrm{Cmcm}$ structure at $125 \mathrm{GPa}$, close to the center of its stability range (see Fig. 7). First, computing the dynamical matrices on a $(6,6,6)$ $q$-point grid, we observe an imaginary TA branch along the $\Gamma-Z$ direction, having a minimum at the zone-boundary $Z$ point. The equivalent calculation of the phonon dispersion curves for the unmodulated $\mathrm{Cmcm}$ structure using a $(4,4,2)$ uniform $q$-point grid (Fig. 9) shows three imaginary frequencies at $\Gamma$ associated with the modes $B_{2 u}, B_{1 u}$, and $A_{g}$. These modes involve different displacements of the $y$ parameter of $\mathrm{P}(4 a)$ and $\mathrm{P}(4 c)$ atoms. In particular, the $A_{g}$ mode corresponds to the proposed modulation of the approximant where only the $y$ parameter of $\mathrm{P}(4 c)$ deviates from 0 . Interestingly, a minimum of the soft phonon curve appears along the $\Gamma-Z$ direction in accordance with the group-theoretical analysis. The explanation comes from the fact that $c_{C m c m}^{*}=\frac{1}{4} c_{C m m m}^{*}$ meaning that the $(4,4,2)$ mesh is denser along the $c$ direction
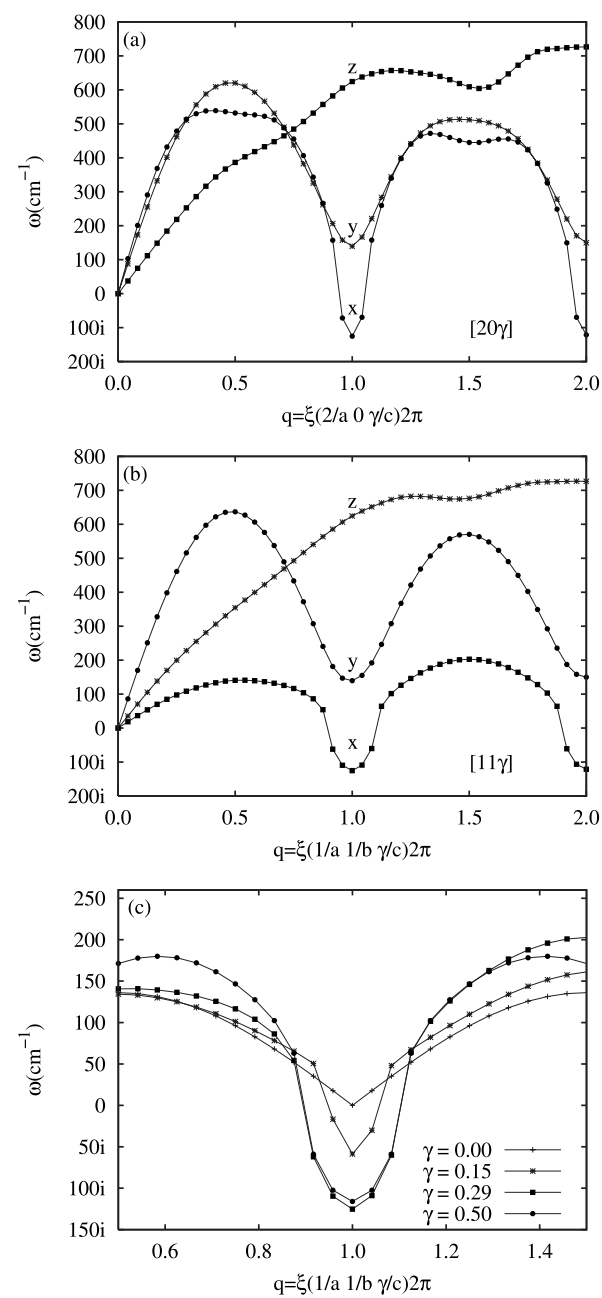

FIG. 10. Calculated phonon dispersions of the Cmmm structure along (a) the $[20 \gamma]$ and (b) the $[11 \gamma]$ directions with $\gamma=0.29$. Figure $10(\mathrm{c})$ shows the soft phonon along the $[11 \gamma]$ direction for several values of $\gamma$.

than the $(6,6,6)$ mesh used in the two-atom $\mathrm{Cmmm}$ structure. This emphasizes the importance of using a denser mesh to detect the incommensurability.

Thus we carried out a much more accurate interpolation of the TA branch of the Cmmm structure with a denser mesh of $q$-points $(2 \times 2 \times 48)$ (Fig. 9). This reveals that the minimum is, in fact, located at 0.29 of the $\Gamma-Z$ distance and related to the incommensuration of the phase.

A clearer picture comes from the analysis of the phonon dispersion curves along the $[11 \gamma]$ and $[20 \gamma]$ directions of the average $\mathrm{Cmmm}$ structure, motivated by the presence of the (1101) and (2001) satellite Bragg peaks in the diffraction pattern of the P-IV phase (see Fig. 2). We clearly observe (see Fig. 10) the imaginary acoustic phonon associated with the $x$ direction of the average $\mathrm{Cmmm}$ structure (corresponding to the $y$ direction of the $\mathrm{Cmcm}$ approximant structure). In addition, calculations of the phonon dispersion curves for several values of $\gamma$ along the direction $[11 \gamma]$ indicate that the minimum of the soft phonon corresponds to the previously calculated $\gamma$ value. The fact that the calculated $\gamma$ value is bigger than the experimental value may be related to the 


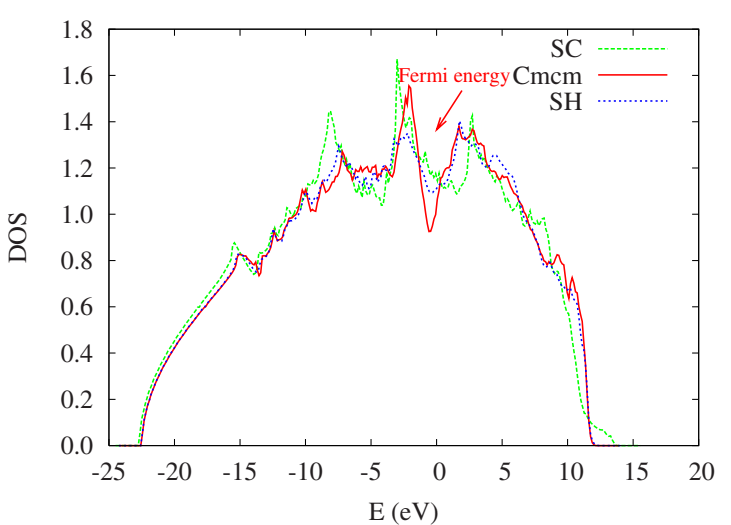

FIG. 11. (Color online) Calculated density of electronic states for $\mathrm{SC}, \mathrm{SH}$, and modulated phosphorus at $150 \mathrm{GPa}$, showing the opening of a pseudogap at $E_{F}$ by the modulation.

larger theoretical $c$ axis (smaller reciprocal $c^{*}$ axis) as a consequence of the GGA approximation.

\section{Electronic density of states}

The calculations (see Fig. 11) clearly show that the main difference in the electronic density of states for the P-IV phase (in the $\mathrm{Cmcm}$ commensurate approximant) compared to the SC and SH phases is a pronounced dip at the Fermi energy. Although the density of states appears free-electronlike at low $\mathrm{E}$, in the total-energy calculation the absolute value of the eigenenergies depends on the background field, and cannot be directly related to a wave vector.

To obtain a deeper understanding of the FSBZ interactions, the electronic band structures of the modulated $\mathrm{Cmcm}$ approximant and the simple cubic structure (in the $\mathrm{Cmcm}$ setting) at the same volume (corresponding to $125 \mathrm{GPa}$ for the Cmcm structure) are calculated (Fig. 12). We find an energy band opening along the SR $\left(\frac{1}{2}, \frac{1}{2}, z\right)$ and TY $(1,0, z)$ directions. These two directions are parallel to the reciprocal $c^{*}$ axis, consistent with the soft phonons (Fig. 10) $(q=2 k)$. In a free-electron picture we expect $2 k_{\mathrm{F}}$ to be equal to a soft phonon and corresponding to a band gap opening and a diffraction peak. In our case, the $2 k_{\mathrm{F}}$ vector is around $5 \AA$. The only feature matching this in the diffraction pattern is the (2011) peak, with a range of other smaller peaks lying slightly below $2 k_{\mathrm{F}}$ as is common in other simple metals. ${ }^{4} \mathrm{In}$ the folded zone picture $(201 \overline{1})$ maps back onto $(001 \overline{1})$, i.e., the direction of the incommensuration in 3D. Thus, in this picture, the incommensurate wave vector comes from electron-phonon coupling at $q=(201 \bar{\gamma})$, which of course leads to exactly the same atomic displacements as the $(00 \gamma)$ phonon. The band structure in the other directions remains metallic and contributes to the residual finite density of states at the Fermi energy.

\section{Electron localization function}

Corroboration of the underlying chemical significance of these pressure-induced transitions was sought in the chemical bonding of the different phases as accounted for by the ELF. This function is a local measure of electron localization
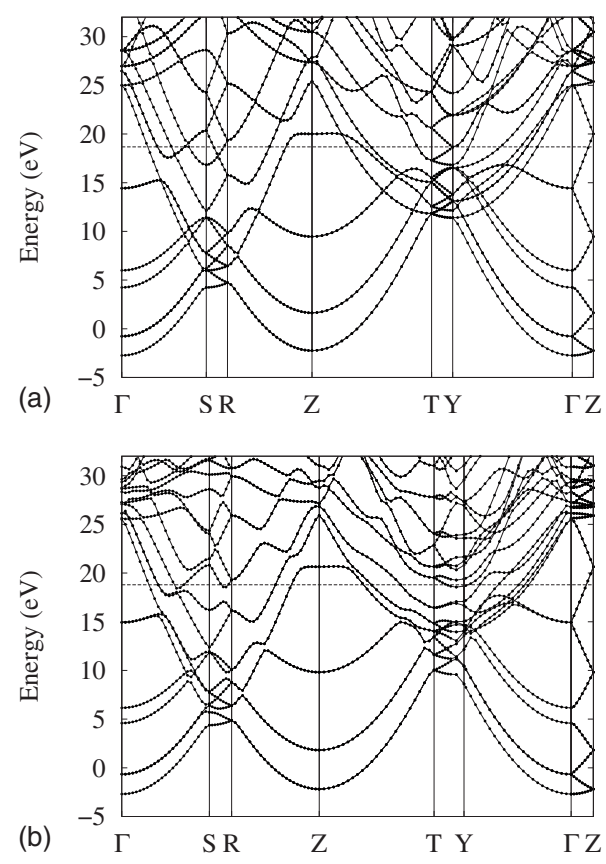

FIG. 12. Electronic band structure of (a) the SC, and (b) the $\mathrm{Cmcm}$ commensurate approximant of P-IV, at $125 \mathrm{GPa}$. Both calculations were performed using the $\mathrm{Cmcm}$ structure.

within a system based on Pauli's principle. It approaches 1 in those regions of space where localization is high, and tends to a value of 0.5 when the density distribution approaches homogeneity. The electron localization function beautifully recovers the opening of the pseudogap in the transition from the simple cubic to the $\mathrm{Cmcm}$ phase. Figure 13(a) shows how the 0.6 ELF isosurfaces in the SC structure (in yellow) are connected across the whole crystal, consistent with its metallic nature. Moreover, this metallic behavior is also manifested across the (001) planes where the valence basins (blue regions) are connected with a homogeneous ELF.

It is also notable that the ELF maxima found in the SC phase are connected by a saddle point with a very high value of ELF (0.72), that is indicative of the pairing of electrons in P-III within the static approximation $(T=0 \mathrm{~K})$, probably related to its superconductivity at low temperatures. The analysis of the behavior of the ELF topology under pressure reveals that two localization maxima along the P-P bonding direction progressively approach each other until they collapse at the transition pressure. Figure 13(c) shows the appearance of the "covalent" bonds (in blue) in the $\mathrm{Cmcm}$ phase, as well as the deformation of the 0.6 isosurfaces (in yellow) into more localized domains along the bonding directions and its partial rupture along the $c$ axis due to the modulation [Fig. 13(d)]. This topological catastrophe gives a proof in direct space of the relationship between the localization of electrons and the opening of the pseudogap stabilizing the $\mathrm{Cmcm}$ phase. The last step of the studied transition sequence, which is the simple hexagonal phase, is shown in Fig. 13(b), whereby the ELF shows the culmination of the stratification in metallic $a b$ layers connected by separated covalent bonds along the $c$ axis. 


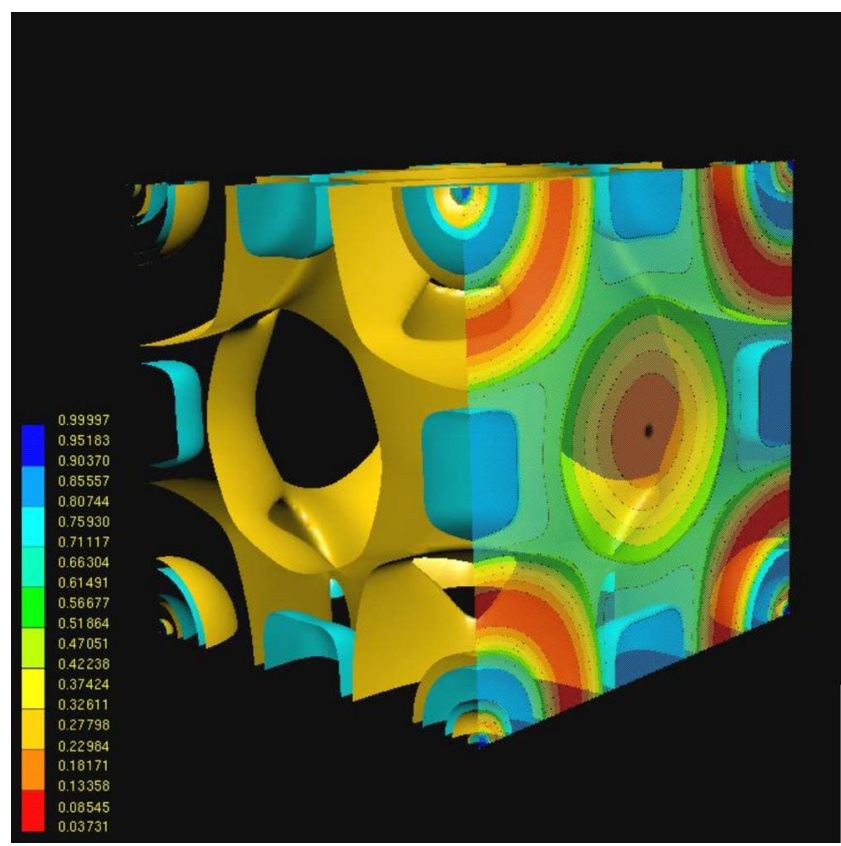

(a)

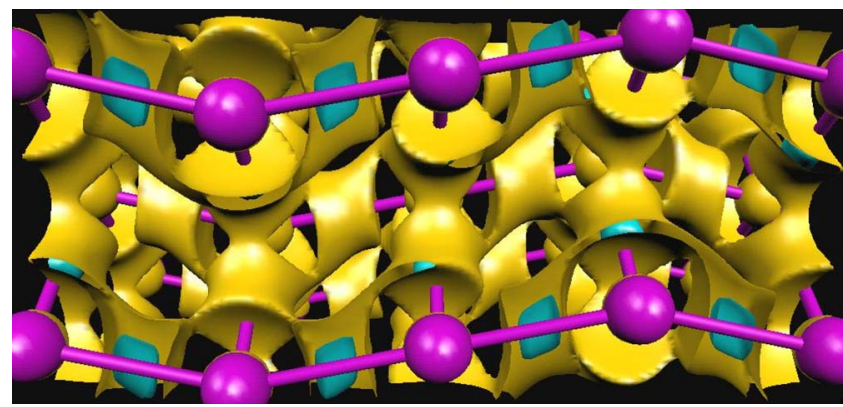

(c)

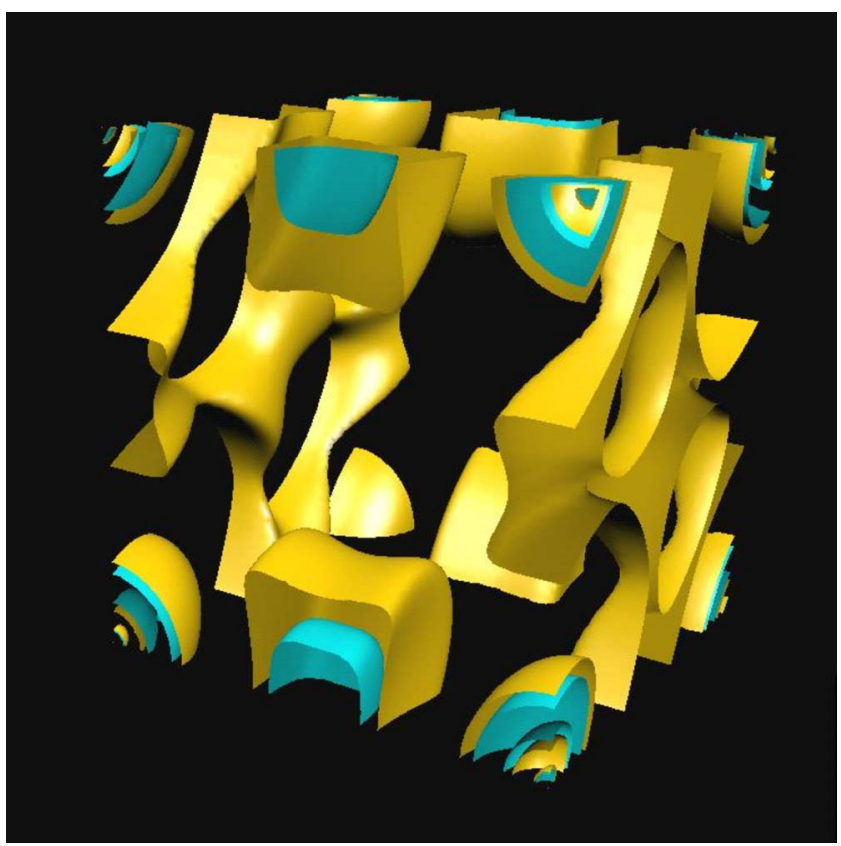

(b)

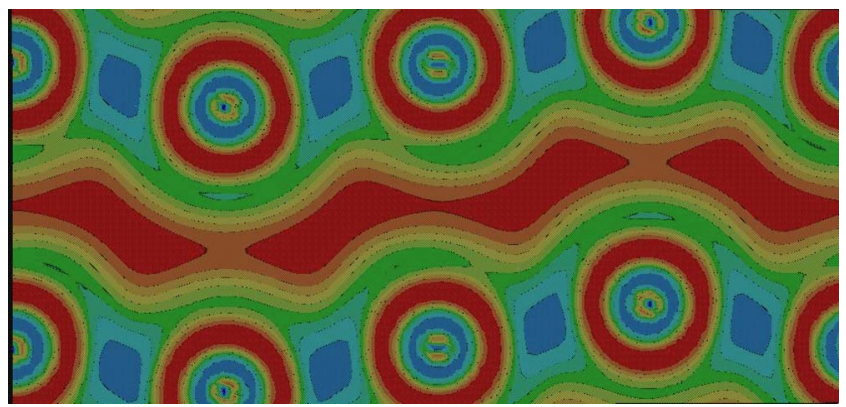

(d)

FIG. 13. (Color online) Isosurfaces and ELF profiles of (a) the SC, (b) the SH and (c and d) the Cmcm structures. The represented isosurfaces in (a), (b) and (c) correspond to the values 0.6 (yellow) and 0.8 (blue), and the ELF scale varies between 0 (red) and 1 (dark blue). (a) Isosurfaces and ELF across the (001) plane of the SC phase. (b) Isosurfaces of SH phase. (c) Isosurfaces of the $\mathrm{Cmcm}$ structure. The magenta spheres show the location of the phosphorus atoms. (d) ELF modulation along the $c$ axis of the $C m c m$ phase.

\section{CONCLUSIONS}

Detailed experimental work has revealed details of the pressure evolution of the incommensurate P-IV structure, and fully confirms the structural solution of Fujihisa et al. Excellent agreement between DFT calculations and experiment gives us confidence that the electronic structure is correctly described, and can form the basis for an explanation of the phase stability. Further, we can assume theoreticallypredicted phonons, pressure dependence of the lattice parameters and modulation amplitudes are correct.

The simple cubic-Cmcm transition is likely to occur continuously, via a coupled phonon-lattice parameter distortion. Although the simple hexagonal phase is also connected to Cmcm by a group-subgroup relation, the phonon associated with this phase transformation does not soften, thus the transition is likely to be more strongly first order.

The incommensuration is revealed by an unstable phonon in the approximant $\mathrm{Cmcm}$ phase. The softening of this mode appears to be due to a Fermi-surface effect, opening a pseudogap in the free-electron-like density of states. This opening is related to the progressive electron localization along the $c$ axis as depicted by the evolution of the electron localization function under pressure. As a consequence, the softening is only revealed by well-converged phonon calculations with a very high $k$-point sampling density. Poor $k$-point sampling will not properly describe the Fermi surface. In the actual transition, the soft phonon is coupled to a small lattice distortion.

The pseudogap opens along the SR and TY branches of the P-IV which, along with localization effects in the electronic structure, account for the sharply increased resistivity which accompanies the onset of the P-IV phase.

In summary, we have presented a detailed and fully consistent experimental and theoretical picture of P-IV, relating the structural properties of the atoms to the minimization of the electronic energy, with the incommensurization ultimately caused by Fermi-surface effects. 


\section{ACKNOWLEDGMENTS}

We thank Ulrich Schwarz and Aron Wosylus of the MaxPlanck-Institut für Chemische Physik fester Stoffe in Dresden for supplying the sample of black phosphorus. This work was supported by grants from EPSRC, and facilities provided by the ESRF. J.C.G. thanks financial support from the Spanish MEC and FEDER program under Project No. MAT2006-13548-C02-02 and the Spanish MALTAConsolider-Ingenio-2010 (Grant No. CSD2007-00045).
*Corresponding author: mim@ph.ed.ac.uk

${ }^{1}$ M. I. McMahon and R. J. Nelmes, Chem. Soc. Rev. 35, 943 (2006).

${ }^{2}$ M. I. McMahon and R. J. Nelmes, Z. Kristallogr. 219, 742 (2004).

${ }^{3}$ G. J. Ackland and I. R. MacLeod, New J. Phys. 16, S2629 (2004).

${ }^{4}$ G. J. Ackland, Rep. Prog. Phys. 64, 483 (2001).

${ }^{5}$ S. Falconi and G. J. Ackland, Phys. Rev. B 73, 184204 (2006).

${ }^{6}$ J.-Y. Raty, E. Schwegler, and S. A. Bonev, Nature (London) 449, 448 (2007).

${ }^{7}$ Y. Akahama, M. Kobayashi, and H. Kawamura, Phys. Rev. B 59, 8520 (1999).

${ }^{8}$ R. Ahuja, Phys. Status Solidi B 235, 282 (2003).

${ }^{9}$ F. J. H. Ehlers and N. E. Christensen, Phys. Rev. B 69, 214112 (2004).

${ }^{10}$ A. S. Mikhaylushkin, S. I. Simak, B. Johansson, and U. Haussermann, Phys. Rev. B 76, 092103 (2007).

${ }^{11}$ T. Ishikawa, H. Nagara, K. Kusakabe, and N. Suzuki, Phys. Rev. Lett. 96, 095502 (2006).

${ }^{12}$ H. Fujihisa, Y. Akahama, H. Kawamura, Y. Ohishi, Y. Gotoh, H. Yamawaki, M. Sakashita, S. Takeya, and K. Honda, Phys. Rev. Lett. 98, 175501 (2007).

${ }^{13}$ C. Hejny and M. I. McMahon, Phys. Rev. Lett. 91, 215502 (2003).

${ }^{14}$ M. Hanfland, K. Syassen, and J. Kohler, J. Appl. Phys. 91, 4143 (2002).

${ }^{15}$ A. P. Hammersley, S. O. Svensson, M. Hanfland, A. N. Fitch, and D. Hausermann, High Press. Res. 14, 235 (1996).
${ }^{16}$ V. Petricek and M. Dusek, The Crystallographic Computing System JANA2000 (Institute of Physics, Praha, Czech Republic, 2000).

${ }^{17}$ M. I. McMahon, C. Hejny, J. S. Loveday, L. F. Lundegaard, and M. Hanfland, Phys. Rev. B 70, 054101 (2004).

${ }^{18}$ G. Kresse and J. Furthmuller, Phys. Rev. B 54, 11169 (1996).

${ }^{19}$ J. P. Perdew and Y. Wang, Phys. Rev. B 45, 13244 (1992).

${ }^{20}$ G. Kresse and D. Joubert, Phys. Rev. B 59, 1758 (1999).

${ }^{21}$ H. J. Monkhorst and J. D. Pack, Phys. Rev. B 13, 5188 (1976).

${ }^{22}$ M. A. Blanco, E. Francisco, and V. Luaña, Comput. Phys. Commun. 158, 57 (2004).

${ }^{23}$ S. Baroni, S. de Gironcoli, A. Dal Corso, and P. Giannozzi, Rev. Mod. Phys. 73, 515 (2001).

${ }^{24}$ The QUANTUM-ESPRESSO code is available at http:// www.quantum-espresso.org

${ }^{25}$ D. Vanderbilt, Phys. Rev. B 41, 7892 (1990).

${ }^{26}$ A. D. Becke and K. E. Edgecombe, J. Chem. Phys. 92, 5397 (1990).

${ }^{27}$ B. Silvi and A. Savin, Nature (London) 371, 683 (1994).

${ }^{28}$ J. Contreras-García, A. Martín Pendás, B. Silvi, and J. M. Recio, J. Phys. Chem. Solids (to be published).

${ }^{29}$ V. R. Saunders, R. Dovesi, C. Roetti, M. Causá, N. M. Harrison, R. Orlando, and C. M. Zicovich-Wilson, CRYSTAL98 User's Manual (University of Torino, Torino, 1998).

${ }^{30}$ Y. Akahama, H. Kawamura, S. Carlson, T. Le Bihan, and D. Haüsermann, Phys. Rev. B 61, 3139 (2000).

${ }^{31}$ H. T. Stokes, B. J. Campbell, and D. M. Hatch, Acta Crystallogr., Sect. A: Found. Crystallogr. 63, 365 (2007). 